\title{
Effects of a Chinese medical herbs complex on cellular immunity and toxicity-related conditions of breast cancer patients
}

\author{
S. R. Zhuang ${ }^{1,2}$, H. F. Chiu ${ }^{3}$, S. L. Chen ${ }^{2}$, J. H. Tsai ${ }^{1}$, M. Y. Lee ${ }^{4}$, H. S. Lee ${ }^{2}$, Y. C. Shen ${ }^{2}$, \\ Y. Y. Yan ${ }^{2}$, G. T. Shane ${ }^{5}$ and C.-K. Wang ${ }^{2 *}$ \\ ${ }^{1}$ Department of Nutrition, Chung Shan Medical University Hospital, No. 110, Section 1, Jianguo North Road, \\ Taichung 40201, Taiwan, ROC \\ ${ }^{2}$ School of Nutrition, College of Health Care and Management, Chung Shan Medical University, No. 110, Section 1, \\ Jianguo North Road, Taichung 40201, Taiwan, ROC \\ ${ }^{3}$ Department of Integrated Chinese and Western Medicine, Chung Shan Medical University Hospital, No. 110, Section 1, \\ Jianguo North Road, Taichung 40201, Taiwan, ROC \\ ${ }^{4}$ Department of Statistics and Informatics Science, Providence University, 200 Chung Chi Road, Taichung 43301, \\ Taiwan, $R O C$ \\ ${ }^{5}$ Medigreen Biotechnology Corporation, 5F, No. 611, Section 1, Wanshou Road, Gueishan Shiang, Taoyuan 333, \\ Taiwan, ROC \\ (Received 25 August 2010 - Revised 24 May 2011 - Accepted 26 May 2011 - First published online 25 August 2011)
}

\begin{abstract}
Rose geranium (Pelargonium graveolens, Geraniaceae) has anti-cancer and anti-inflammatory properties, and promotes wound healing. Similarly, Ganoderma tsugae (Ganodermataceae), Codonopsis pilosula (Campanulaceae) and Angelica sinensis (Apiaceae) are traditional Chinese herbs associated with immunomodulatory functions. In the present study, a randomised, double-blind, placebo-controlled study was conducted to examine whether the Chinese medicinal herb complex, RG-CMH, which represents a mixture of rose geranium and extracts of G. tsugae, C. pilosula and A. sinensis, can improve the immune cell count of cancer patients receiving chemotherapy and/or radiotherapy to prevent leucopenia and immune impairment that usually occurs during cancer therapy. A total of fifty-eight breast cancer patients who received chemotherapy or radiotherapy were enrolled. Immune cell levels in patient serum were determined before, and following, 6 weeks of cancer treatment for patients receiving either an RG-CMH or a placebo. Administration of RG-CMH was associated with a significant reduction in levels of leucocytes from $31.5 \%$ for the placebo group to $13.4 \%$ for the RG-CMH group. Similarly, levels of neutrophils significantly decreased from $35.6 \%$ for the placebo group to $11.0 \%$ for the RG-CMH group. RG-CMH intervention was also associated with a decrease in levels of $\mathrm{T}$ cells, helper $\mathrm{T}$ cells, cytotoxic $\mathrm{T}$ cells and natural killer cells compared with the placebo group. However, these differences between the two groups were not statistically significant. In conclusion, administration of RG-CMH to patients receiving chemotherapy/radiotherapy may have the capacity to delay, or ease, the reduction in levels of leucocytes and neutrophils that are experienced by patients during cancer treatment.
\end{abstract}

Key words: Breast cancer: Chemotherapy: Radiotherapy: Chinese medical herbs: Immune cell counts

In 2006, breast cancer was the fourth leading cause of death in Taiwan and more than 5200 new cases of breast cancer had been diagnosed 2 years earlier. With such a high rate of incidence and prevalence, breast cancer remains an important public health issue in Taiwan. For high-risk cancer patients, they typically undergo surgery, followed by systemic chemotherapy and/or local radiotherapy as a standard adjuvant prophylaxis against disseminated tumour cells ${ }^{(1)}$. As a result, the combination of the cancer itself, as well as treatments including surgery, chemotherapy and/or radiation, impairs a patient's immunity ${ }^{(2)}$. For example, chemotherapy has cytostatic or cytotoxic effects on rapidly dividing cells, leading to adverse side effects. These side effects can include leucopenia, neutropenia and thrombocytopenia, which are attributable to bone marrow suppression, as well as suppression of the proliferation or function of lymphocyte subsets. Moreover, overall $\mathrm{T}$ cell levels have been found to be significantly lower in patients who received adjuvant systemic therapy $v$. patients

Abbreviations: AE, adverse event; NK, natural killer.

*Corresponding author: C.-K. Wang, fax +886 423248192 , email wck@csmu.edu.tw 
with primary breast cancer or normal healthy donors ${ }^{(3)}$. The immune system may also play an important role in mediating tumour growth and metastasis ${ }^{(4,5)}$. Accordingly, cell-mediated immunity to tumour-associated antigens has been shown to be a better predictor of patient survival than tumour stage, grade and lymph node status ${ }^{(4)}$.

Many patients undergoing cancer therapy consider complementary and alternative medicine options, which can include vitamins, Chinese medical herbs or other biological products $^{(6)}$. Usually, these products are taken to reduce side effects and organ toxicity, to protect and stimulate immunity or to prevent further development of additional cancers or recurrence ${ }^{(7,8)}$. However, scientific evidence regarding the efficacy and safety of herbs and nutritional supplements remains limited.

Ganoderma tsugae (also known as Lingzhi), Codonopsis pilosula (also known as Dang shen) and Angelica sinensis (also known as Dong quai) are often used in Chinese medicine, and are a source of food in Taiwan. Moreover, Lingzhi, which was recorded in traditional Chinese medicine as early as 2000 years ago, has been used to cure many diseases and to promote human vitality. Correspondingly, many therapeutic effects have been associated with mushrooms of diverse Lingzhi species, and these include immunomodulatory, antitumour, hepatoprotective, antioxidant, cholesterol-lowering, hypoglycaemia-inducing and appetite-suppressing activities $^{(9)}$. Moreover, these observed therapeutic effects have been attributed to triterpenoid, polysaccharide and/or glycoprotein content of Lingzhi, although the exact mechanisms responsible for the observed biological activities have not been determined. Nevertheless, Lingzhi has traditionally been consumed as a luxurious functional food supplement in Chinese society.

C. pilosula belongs to the Campanulaceae family, and its root is commonly used in Chinese medicine. It has been used for the treatment of dyspepsia, poor appetite, fatigue and psychoneurosis. More recently, it has also been shown to mediate immunomodulatory activities of mouse splenocytes $^{(10)}$. A. sinensis Diels is another well-known Chinese herbal medicine that has been used for the treatment of various diseases and as a tonic medicine for thousands of years, especially for gynaecological diseases ${ }^{(11)}$. It has also been used to treat anaemia due to enhanced haematopoiesis by stimulating the secretion of haematopoietic growth factors $^{(12)}$.

Rose geranium (Pelargonium graveolens) is a multi-harvest, high-value, aromatic plant cultivated for its essential oil that is widely used in the fragrance industry, for aromatherapy and for extraction of commercial rhodinol (a mixture of linalool, citronellol and geraniol). Essential oils from rose geranium have also been associated with strong antioxidant effects ${ }^{(13)}$. Therefore, in the present study, the Chinese medicinal herb complex, RG-CMH, which includes extracts of rose geranium, G. tsugae, C. pilosula and A. sinensis, was evaluated in a randomised, double-blind, placebo-controlled study of fifty-eight patients with breast cancer and those that were prescribed chemotherapy or radiotherapy. Immune cell counts before, and 6 weeks after, treatment were assayed.

\section{Experimental methods}

\section{Subjects}

The present study was conducted according to the guidelines laid down in the Declaration of Helsinki and all procedures involving human patients were approved by the Institutional Review Board of the Chung Shan Medical University Hospital. Written informed consent was obtained from all the patients.

A total of sixty-six patients $\geq 18$ years of age with stage I-IV breast cancer (according to the International Union Against Cancer TNM classification of malignant tumours) were treated with surgery followed by chemotherapy or radiotherapy at the Chung Shan Medical University Hospital (Taichung, Taiwan) and were then enrolled in the present study. Exclusion criteria for participation included in situ breast carcinoma patients, terminally ill patients, renal failure, congestive heart failure or hepatic failure, as well as intolerance or poor compliance with herbal medicine.

\section{Study design}

Between December 2004 and November 2005, a randomised, placebo-controlled study was conducted. A physical examination was performed at the beginning of the study, which recorded body height, body weight, vital signs, blood pressure and anamnesis. Patients confirmed that other systemic diseases had not been diagnosed, and that adverse effects had not previously been experienced following the use of any herbal medicines. Enrolled patients were randomly assigned to receive either nine capsules of $\mathrm{RG}-\mathrm{CMH}$ (experimental group) or placebo (control group) each day, and patients received medicinal herb treatment and chemotherapy concomitantly. Patients who received chemotherapy by injection were required to have an overnight stay in the hospital, followed by a 3-week recovery period. The treatment period for each patient was 6 weeks (which included two chemotherapy cycles). Blood samples were collected before the first/ next chemotherapy cycle for the two groups for biochemical analysis. The patients were also requested to visit their physician every $7 \mathrm{~d}$ to have leucocytes counts monitored, and to return patient diaries. Tumour location and staging details were obtained from patient medical records.

\section{Compliance control}

Compliance control was performed on the basis of empty packaging and unused investigational products documented for each patient. The compliance of each patient was also evaluated based on the daily feedback recorded and their presence at assigned examination appointments.

\section{Efficacy evaluation}

Primary efficacy criterion was defined as a reduction in levels of leucocytes, neutrophils, lymphocytes and lymphocyte subsets, and a decrease in the number of adverse events (AE) related to chemotherapy. Venous blood samples were obtained to assay the complete blood count and each patient's 
immunity status was analysed before, and following, 6 weeks of treatment. Erythrocytes, Hb, haematocrit, platelets, leucocytes, neutrophils and lymphocytes were evaluated using an automated blood analyser. Lymphocyte subsets (e.g. T cells, helper $\mathrm{T}$ cells, cytotoxic $\mathrm{T}$ cells), B cells and natural killer (NK) cells were evaluated using flow cytometry. Peripheral blood specimens were labelled with Trucount reagent (BD Biosciences, San Jose, CA, USA) and incubated with antibodies to lymphocyte subsets (e.g. $\mathrm{CD}^{+}, \mathrm{CD}^{+} / \mathrm{CD}^{+}$and $\mathrm{CD} 3^{+} /$ $\left.\mathrm{CD}^{+}\right)$, B cells $\left(\mathrm{CD} 19^{+}\right)$and NK cells $\left(\mathrm{CD} 3^{-} / \mathrm{CD} 16^{+} / \mathrm{CD} 56^{+}\right)$ for $15 \mathrm{~min}$ at room temperature, were then subjected to erythrocyte lysis. Four-colour flow cytometric immunophenotyping was performed using a FACSCalibur flow cytometer and Multiset software (BD Biosciences). At least 16500 ungated events were collected and were expressed as a percentage of the total lymphocyte population detected. Both the percentage and absolute count were obtained for each subtype.

Common Terminology Criteria for Adverse Events (CTCAE v3.0) were used (http://ctep.cancer.gov/reporting/ctc.html) to assess AE related to chemotherapy. An AE was defined as any unfavourable and unintended sign (including an abnormal laboratory finding), symptom or disease temporally associated with the use of a medical treatment or procedure that may or may not be considered a medical treatment or procedure. The CTCAE v3.0 displays grades $1-5$ with unique clinical descriptions of the severity associated with each $\mathrm{AE}$ grade. For example, grade 1 represents a mild $\mathrm{AE}$, grade 2 a moderate $\mathrm{AE}$, grade 3 is a severe $\mathrm{AE}$, grade 4 is a life-threatening or disabling $\mathrm{AE}$ and grade 5 is a death-related $\mathrm{AE}$. Information regarding $\mathrm{AE}$ related to cancer treatment was obtained from patient medical records. AE included anorexia, vomiting, diarrhoea, distension/abdominal bloating, changes in hearing, and changes in saliva and/or the salivary gland.

\section{Test material}

A placebo capsule containing maize starch $(115.6 \mathrm{mg})$, magnesium stearate $(2.5 \mathrm{mg}), \beta$-cyclodextrin $(363.7 \mathrm{mg})$ and caramel $(18.2 \mathrm{mg})$ was administered to the control group. In contrast, the experimental group received an RG-CMH capsule that contained $A$ sinensis extract $(64.5 \mathrm{mg}), C$. pilosula extract $(27 \cdot 1 \mathrm{mg})$, G. tsugae extract $(3.0 \mathrm{mg})$, rose geranium power $(273.6 \mathrm{mg})$, maize starch $(129.3 \mathrm{mg})$ and magnesium stearate ( $2.5 \mathrm{mg}$ ). Liquid extracts of G. tsugae, C. pilosula, and $A$ sinensis were obtained following extraction with boiling water $(\mathrm{v} / \mathrm{w}$ 10) for $1 \mathrm{~h}$. After the extracts were freeze-dried, levels of ferulic acid were detected. Compared with the placebo capsules, RG-CMH capsules were standardised according to the level of ferulic acid detected (e.g. 0.12 mg), and all capsules were made by Sun Ten Pharmaceutical Company Limited (Taoyuan, Taiwan), and provided by Medigreen Biotechnology Corporation. Each patient was required to consume nine capsules per $\mathrm{d}$.

\section{Statistical analysis}

All analyses were carried out in accordance with current clinical practice guidelines and were applied to all variables. Biochemical data are presented as the means and standard deviation. ANCOVA were also applied to erythrocytes, $\mathrm{Hb}$, haematocrit, and platelet counts were determined before, and following, 6 weeks of treatment for both the experimental and the placebo groups. The dependent Student's $t$ test was used to compare differences within the same group, and an independent $t$ test was used to compare differences between the biochemical analyses performed for the control and experimental groups. Statistical analyses of toxicity related to chemotherapy/radiotherapy for the control and experimental groups were also determined using the $\chi^{2}$ test. A $P$ value $<0.05$ was considered statistically significant. The Statistics Package for the Social Sciences (SPSS) statistical software package version 10.0 (Chicago, IL, USA) was used for all statistical analyses performed.

\section{Results}

\section{Study group characteristics}

A total of sixty-six subjects were enrolled in the present study, and were randomised into two groups. Patients in the experimental group ( $n$ 33) were assigned to take nine capsules containing RG-CMH a day, while patients in the control group ( $n$ 33) were assigned nine placebo capsules each day. Out of sixty-six patients, fifty-eight completed this 6-week study; two from the experimental group and four from the control group withdrew from the study due to changes in their medical therapy programmes; one control patient withdrew due to the development of a mild rash, and another control patient due to intolerance of the capsule flavour. Patient characteristics evaluated for both the experimental group ( $n$ 31) and the control group ( $n$ 27) at the start of the study are presented in Table 1 . Treatment regimens received by patients of the control group included: one cycle of chemical therapy ( $n$ 25), two cycles of chemotherapy $(n$ 1) and radiotherapy $(n 1)$. For the experimental group, twenty-nine patients underwent one cycle of chemical therapy, one patient underwent two cycles of chemotherapy and one patient underwent radiotherapy. There were no significant differences in any of the variables compared between the two patient groups at the start of the study (Table 1).

\section{Compliance control}

All fifty-eight patients remained in the study for the required duration, returned the empty packaging and unused investigational product, and filled out their diaries carefully. For both groups, the ratio of empty packaging to total packaging was $99.9 \%$.

\section{Administration of RG-CMH affected haematological indicators}

The mean platelet concentration for the control group was $247 \cdot 11$ (SD 59.21) $\times 10^{3} / \mathrm{mm}^{3}$ at the start of the study, yet decreased significantly to 227.67 (SD 52.97$) \times 10^{3} / \mathrm{mm}^{3}$ by week 6 ( $P=0 \cdot 047$; Table 2$)$. In contrast, there was no difference in the platelet concentrations detected for the experimental 
Table 1. Baseline characteristics for this cohort

(Mean values and standard deviations or numerical value for each group)

\begin{tabular}{|c|c|c|c|c|c|}
\hline \multirow[b]{2}{*}{ Variable } & \multicolumn{2}{|c|}{$\begin{array}{l}\text { Control group } \\
\quad(n 27)\end{array}$} & \multicolumn{2}{|c|}{$\begin{array}{l}\text { Experimental } \\
\text { group }(n 31)\end{array}$} & \multirow[b]{2}{*}{$P^{*}$} \\
\hline & Mean & SD & Mean & SD & \\
\hline Age (years) & 53.5 & $10 \cdot 3$ & 49.9 & 8.6 & $0.193 \dagger$ \\
\hline Height $(\mathrm{cm})$ & 154.7 & 4.9 & $156 \cdot 4$ & $5 \cdot 0$ & $0.128 \dagger$ \\
\hline Weight (kg) & 57.9 & $10 \cdot 4$ & $59 \cdot 2$ & 8.9 & $0.738 \dagger$ \\
\hline BMI $\left(\mathrm{kg} / \mathrm{m}^{2}\right)$ & 24.5 & 4.5 & $24 \cdot 1$ & $3 \cdot 2$ & $0.328 \dagger$ \\
\hline Female patients & \multicolumn{2}{|c|}{27} & \multicolumn{2}{|c|}{31} & $0.346 \ddagger$ \\
\hline Disease stage & & & & & $0.433 \ddagger$ \\
\hline 1 & \multicolumn{2}{|c|}{3} & \multicolumn{2}{|c|}{5} & \\
\hline II & \multicolumn{2}{|c|}{16} & \multicolumn{2}{|c|}{13} & \\
\hline III & \multicolumn{2}{|c|}{5} & \multicolumn{2}{|c|}{11} & \\
\hline IV & \multicolumn{2}{|c|}{2} & \multicolumn{2}{|c|}{2} & \\
\hline Unknown & \multicolumn{2}{|c|}{1} & \multicolumn{2}{|c|}{0} & \\
\hline Treatment received & & & & & $0.879 \ddagger$ \\
\hline Injections§ & \multicolumn{2}{|c|}{13} & \multicolumn{2}{|c|}{14} & \\
\hline Orall| & \multicolumn{2}{|c|}{5} & \multicolumn{2}{|c|}{4} & \\
\hline Injections + oral & \multirow{2}{*}{\multicolumn{2}{|c|}{$\begin{array}{l}8 \\
1\end{array}$}} & \multirow{2}{*}{\multicolumn{2}{|c|}{12}} & \\
\hline Radiotherapy & & & & 1 & \\
\hline
\end{tabular}

* There was no significant difference in sex, age, height, weight, BMI, type of carcinoma, tumour stage or type of therapy between the two groups.

$\dagger$ According to $t$ test between control and experimental groups.

$\ddagger$ According to $\chi^{2}$ test between control and experimental groups.

$\S$ Pharmorubicin, endoxan and fluorouracil were injected with dosages determined by body surface area.

II Novofen and UFUR were administered according to body weight.

I Radiotherapy: $57.6 \mathrm{GY} / 32 \mathrm{FX}$ was administered to control group patients and $55.8 \mathrm{GY} / 31 \mathrm{FX}$ was administered to experimental group patients.

group at the start of the study $v$. week 6 . Nevertheless, according to ANCOVA tests (which included adjustments for $\mathrm{Hb}$ and haematocrit baseline values), concentrations of platelets, as well as of erythrocytes, $\mathrm{Hb}$ and haematocrit, showed no significant differences between the two groups between the start of the study and at week 6.

\section{Administration of RG-CMH affected immunological} parameters

Using flow cytometry, we detected a significant decrease in the percentage of leucocytes and neutrophils for the control and experimental groups after 6 weeks of treatment, and this observation was independent of supplement (Table 3). For example, leucocyte levels decreased 31.5 (SD 14.1) \% in the control group, while a 13.4 (SD 20.1)\% decrease was detected in the experimental group after 6 weeks. For neutrophils, the control group exhibited a 35.6 (SD 18.3)\% decrease, and the experimental group exhibited an 11.0 (SD 39.7) \% decrease. In both the cases of change in leucocytes and of neutrophils during the study, the difference between experimental and control groups was statistically significant. In combination, these results demonstrate that a significant reduction in leucocytes and neutrophils accompanied the administration of RG-CMH capsules in this cohort.

For lymphocyte levels, a decrease of 21.9 (SD 20.5) \% and $12 \cdot 1$ (SD 28.8) \% was detected for the control and experimental groups respectively. Similarly, levels of $\mathrm{T}$ lymphocytes were found to decrease 18.3 (SD 20.9) $\%$ and 7.5 (SD 26.1) \%, and helper $\mathrm{T}$ lymphocyte levels decreased 18.7 (SD 20.4) \% and $7 \cdot 1$ (SD 30.3) \% in each case. For cytotoxic T lymphocytes, the control group was associated with a $16 \cdot 2$ (SD 24.0)\% decrease, while the experimental group exhibited a decrease of 4.7 (SD 31.6) \%. Moreover, for both the control and the experimental groups, B lymphocyte levels decreased 33.7 (SD $41 \cdot 3$ ) \% and 33.2 (SD 39.7) \%. Furthermore, levels of NK cells decreased 18.9 (SD 39.1) and 6.4 (SD 58.4)\%. Overall, despite change patterns that were similar to those observed for leucocytes and neutrophils, no significant differences in lymphocytes and lymphocyte subsets were detected for the control group or the experimental group after 6 weeks of treatment.

\section{RG-CMH treatment affected chemotherapy/radiotherapy- related toxicities}

Various toxicity-related conditions due to the chemotherapy/ radiotherapy received by patients in this study were reported (Table 4). For example, anorexia was experienced by all patients in the placebo group (e.g. $81.5 \%$ of patients had grade $1,18.5 \%$ of patients had grade 2 ) by week 6 of treatment. Similarly, anorexia was experienced by all of the experimental

Table 2. Haematology analysis performed $\dagger$ (Mean values and standard deviations)

\begin{tabular}{|c|c|c|c|c|c|c|c|c|}
\hline & \multicolumn{4}{|c|}{ Control group ( $n 27) \ddagger$} & \multicolumn{4}{|c|}{ Experimental group $(n 31) \ddagger$} \\
\hline & \multicolumn{2}{|c|}{ Baseline } & \multicolumn{2}{|c|}{ Week 6} & \multicolumn{2}{|c|}{ Baseline } & \multicolumn{2}{|c|}{ Week 6} \\
\hline & Mean & SD & Mean & SD & Mean & SD & Mean & SD \\
\hline Erythrocytes $\S\left(\times 10^{6} / \mathrm{mm}^{3}\right)$ & $4 \cdot 25^{\mathrm{a}}$ & 0.54 & $4 \cdot 30^{\mathrm{a}}$ & 0.54 & $4 \cdot 11^{\mathrm{a}}$ & 0.45 & $4 \cdot 19^{a}$ & 0.45 \\
\hline $\mathrm{Hb} \S(g / l)$ & $125 \cdot 4^{\mathrm{a} *}$ & $09 \cdot 4$ & $124 \cdot 2^{\mathrm{a}}$ & 09.6 & $117 \cdot 2^{\mathrm{a}}$ & $12 \cdot 6$ & $118 \cdot 3^{a}$ & $12 \cdot 7$ \\
\hline Haematocrit§ (\%) & $38 \cdot 23^{a *}$ & 2.50 & $38 \cdot 26^{a}$ & 2.96 & $36 \cdot 16^{a}$ & 3.39 & $36 \cdot 78^{\mathrm{a}}$ & 3.53 \\
\hline Platelet $\S\left(\times 10^{3} / \mathrm{mm}^{3}\right)$ & $247 \cdot 11^{\mathrm{a}}$ & $59 \cdot 21$ & $227 \cdot 67^{b}$ & 52.97 & $259 \cdot 55^{a}$ & $112 \cdot 30$ & $245 \cdot 32^{\mathrm{a}}$ & $108 \cdot 27$ \\
\hline
\end{tabular}


Table 3. Immunological parameters for the two treatment groups

(Mean values and standard deviations)

\begin{tabular}{|c|c|c|c|c|c|c|c|c|c|c|c|c|c|}
\hline & \multicolumn{6}{|c|}{ Placebo } & \multicolumn{6}{|c|}{$\mathrm{RG}-\mathrm{CMH}$} & \multirow[b]{3}{*}{$P^{*}$} \\
\hline & \multicolumn{2}{|c|}{ Baseline } & \multicolumn{2}{|c|}{ Week 6} & \multicolumn{2}{|c|}{$\%$ Decrease } & \multicolumn{2}{|c|}{ Baseline } & \multicolumn{2}{|c|}{ Week 6} & \multicolumn{2}{|c|}{$\%$ Decrease } & \\
\hline & Mean & SD & Mean & SD & Mean & SD & Mean & SD & Mean & SD & Mean & SD & \\
\hline Leucocytes & 6821 & 1612 & 4576 & 1026 & 31.5 & $14 \cdot 0$ & 6446 & 1822 & 5448 & 1505 & 13.4 & $20 \cdot 1$ & $<0.001$ \\
\hline Neutrophils & 4410 & 1473 & 2703 & 875 & $35 \cdot 6$ & $18 \cdot 3$ & 3947 & 1398 & 3263 & 1103 & $11 \cdot 0$ & 39.7 & 0.005 \\
\hline Total lymphocytes & 1843 & 456 & 1413 & 425 & 21.9 & $20 \cdot 5$ & 1881 & 631 & 1574 & 507 & $12 \cdot 1$ & $28 \cdot 8$ & 0.147 \\
\hline T cellst & 1238 & 315 & 995 & 306 & $18 \cdot 3$ & $20 \cdot 9$ & 1211 & 454 & 1080 & 632 & 7.5 & $26 \cdot 1$ & 0.092 \\
\hline Helper T cellsł & 737 & 272 & 587 & 235 & $18 \cdot 7$ & $20 \cdot 4$ & 693 & 285 & 615 & 239 & $7 \cdot 1$ & $30 \cdot 3$ & 0.098 \\
\hline Cytotoxic T cells§ & 436 & 138 & 363 & 159 & $16 \cdot 2$ & $24 \cdot 0$ & 458 & 311 & 413 & 260 & 4.7 & $31 \cdot 6$ & 0.128 \\
\hline B cells $\|$ & 247 & 125 & 147 & 90 & 33.7 & $41 \cdot 3$ & 248 & 142 & 151 & 152 & $33 \cdot 2$ & $39 \cdot 7$ & 0.964 \\
\hline NK cells & 342 & 155 & 258 & 116 & $18 \cdot 9$ & 39.1 & 395 & 217 & 328 & 191 & $6 \cdot 4$ & 58.4 & 0.350 \\
\hline
\end{tabular}

NK, natural killer.

* The independent $t$ test was used to calculate the percent change for the two groups.

$+\mathrm{CD}^{+}$.

$\ddagger \mathrm{CD}^{+} / \mathrm{CD} 4^{+}$.

$\S \mathrm{CD}^{+} / \mathrm{CD}^{+}$.

$\| \mathrm{CD}^{+} 9^{+}$.

ๆ $\mathrm{CD}^{-} / \mathrm{CD} 16^{+} / \mathrm{CD} 56^{+}$

patients (e.g. 90.3\% patients had grade 1, 9.7\% patients had grade 2$)$ throughout the treatment period. In addition, patients in both groups also experienced vomiting (14.8 and 13.0\% respectively), as well as diarrhoea ( $7 \cdot 1$ and $7 \cdot 5 \%$ respectively) by week 6 . Both groups also reported distension, with $85 \cdot 2 \%$ of the control group experiencing grade 1 distension, $11 \cdot 1 \%$ experiencing grade 2 and $3.7 \%$ experiencing grade 3 distension by week 6 . Similarly, $74 \cdot 2 \%$ of experimental patients suffered from grade 1 distension, and $25.8 \%$ experienced grade 2 distension. No significant differences in the rates of anorexia, vomiting, diarrhoea, distension, hearing or changes in saliva or the salivary gland were found between the two groups. Moreover, there were no significant differences in the toxicity related to chemotherapy/radiotherapy experienced by the two groups.

\section{Discussion}

Cancer patients exposed to chemotherapy/radiotherapy are at higher risk of experiencing therapy-induced toxicity. Adverse side effects can include mucositis, xerostomia, changes in taste, dysphagia, nausea, vomiting, diarrhoea and anorexia, which compromise nutritional status and bodily functions.
Many patients will take complementary and/or alternative medicines to reduce side effects and organ toxicity, to protect and stimulate immunity or to prevent the further development of cancer or recurrences. However, most studies that have investigated side effects of chemotherapy/radiotherapy have used animal models, and only a few studies have involved human clinical trials.

G. tsugae, C. pilosula, A. sinensis and rose geranium are often used in Chinese medicine, and are also a food source in Taiwan. However, research regarding these Chinese herbs and immunity has been limited to in vitro studies. Therefore, the present study is the first to evaluate these Chinese herbs in breast cancer patients in a randomised, controlled study. As a result, administration of $\mathrm{RG}-\mathrm{CMH}$ was found to be associated with a significant reduction in levels of leucocytes and neutrophils (with decreases from 31.5 to $13.4 \%$, and 35.6 to $11.1 \%$ respectively in each case). In comparison, another study of patients that received paclitaxel reported a $60 \%$ decrease in leucocyte levels after 2 weeks of treatment, and a decrease of 24 and $57 \%$ was detected 3 and 4 weeks after the start of treatment $^{(14)}$.

Ganoderma, a traditional Chinese medicine, has recently received considerable attention from both the health care

Table 4. Toxicity-related conditions due to chemotherapy/radiotherapy received by patients*

\begin{tabular}{|c|c|c|c|c|c|c|c|c|c|c|c|c|}
\hline \multirow[b]{3}{*}{ Characteristic } & \multicolumn{4}{|c|}{ Grade 1} & \multicolumn{4}{|c|}{ Grade 2} & \multicolumn{4}{|c|}{ Grade 3} \\
\hline & \multicolumn{2}{|c|}{ Placebo† } & \multicolumn{2}{|c|}{$\mathrm{RG}-\mathrm{CMH} \dagger$} & \multicolumn{2}{|c|}{ Placebo† } & \multicolumn{2}{|c|}{$\mathrm{RG}-\mathrm{CMH} \dagger$} & \multicolumn{2}{|c|}{ Placebo† } & \multicolumn{2}{|c|}{ RG-CMH† } \\
\hline & $\mathrm{T} 1$ & $\mathrm{~T} 2$ & T1 & $\mathrm{T} 2$ & $\mathrm{~T} 1$ & T2 & $\mathrm{T} 1$ & $\mathrm{~T} 2$ & $\mathrm{~T} 1$ & T2 & $\mathrm{T} 1$ & $\mathrm{~T} 2$ \\
\hline Anorexia & 88.9 & 81.5 & $96 \cdot 8$ & $90 \cdot 3$ & $7 \cdot 4$ & $18 \cdot 5$ & $3 \cdot 2$ & 9.7 & $3 \cdot 7$ & 0 & 0 & 0 \\
\hline Vomiting & 3.7 & 0 & 0 & 0 & $3 \cdot 7$ & $11 \cdot 1$ & 3.2 & 6.5 & 0 & 3.7 & 0 & 6.5 \\
\hline Diarrhoea & $8 \cdot 8$ & $7 \cdot 1$ & $5 \cdot 7$ & 7.5 & 0 & 0 & 0 & 0 & 0 & 0 & 2.9 & 0 \\
\hline Distension & $70 \cdot 4$ & $85 \cdot 2$ & $87 \cdot 1$ & $74 \cdot 2$ & $29 \cdot 6$ & $11 \cdot 1$ & $12 \cdot 9$ & $25 \cdot 8$ & 0 & 3.7 & 0 & 0 \\
\hline Hearing & - & - & - & - & 0 & 3.7 & 3.2 & 6.5 & 0 & 0 & 0 & 0 \\
\hline Salivary gland/saliva changes & $29 \cdot 6$ & 44.4 & $29 \cdot 0$ & $29 \cdot 0$ & 7.4 & 3.7 & 6.5 & 3.2 & 0 & 0 & 0 & $3 \cdot 2$ \\
\hline
\end{tabular}

T1, baseline; T2, week 6 .

Values were significantly different: $P<0.05$

* Data listed represent percentages and no adverse event grade 4 conditions reported.

† The $\chi^{2}$ test was used to compare placebo and RG-CMH groups. 
and cancer research communities in Taiwan. For centuries, Ganoderma has been used for medicinal purposes in Asian countries to treat various human diseases, including cancer. Ganoderma lucidum and G. tsugae are the most widely cultivated species of the Ganoderma genus in Taiwan, and both have a long history of being used in folk medicine throughout Asia. Triterpenoids from G. tsugae have been shown to induce apoptosis and arrest the cell cycle in human hepatoma cells $^{(15)}$, while FIBP-gts, a fungal protein from G. tsugae, has been associated with immunomodulatory activity ${ }^{(16)}$. In the latter case, pre-clinical studies have established that the polysaccharide fractions of $G$. tsugae specifically mediate immunomodulatory effects ${ }^{(17-20)}$. In combination, these results demonstrate that $\mathrm{RG}-\mathrm{CMH}$ intervention has the potential to reduce leucopenia.

For approximately $80 \%$ of cancer patients undergoing treatment, neutropenia is a dangerous complication that is often reported $^{(21)}$. However, the exact incidence of neutropenia and the number of neutropenia-related cancer deaths is not clear, with rates of mortality ranging from 5 to $30 \%$ among patients older than 70 years $^{(22)}$. In a study by Tong et al. ${ }^{(14)}$, cancer patients received docetaxel, and after 1 week, untreated levels were detected in $21 \%$ of cases. In comparison, a decrease in neutrophil levels of $35.6 \%$ and $11.0 \%$ for the placebo and RG-CMH groups, respectively, was detected at week 6 in the present study. On the basis of these results, it appears that RG-CMH treatments may delay, or ease, reductions in neutrophil levels during cancer treatment. Furthermore, it has been hypothesised that the maintenance of leucocyte and neutrophil levels associated with RG-CMH is mediated by the polysaccharide of G. tsugae.

NK cells have previously been associated with a spontaneous cytolytic activity that influences unsensitised lymphoid cells. Correspondingly, this process plays an important role in the immune surveillance of malignant tumours $^{(23)}$, and in the immune defence against certain infections, particularly viral infections ${ }^{(24)}$. Women with breast cancer are often at risk for elevated levels of depression and anxiety, as well as reduced levels of NK cells. NK cells are associated with anti-cancer activities that include the lysis of tumour and virus-infected cells, as well as a capacity for monitoring and targeting neoplastic (both new and abnormal) growth $^{(25,26)}$. Correspondingly, higher levels of NK cell activity in patients with breast cancer were associated with a lower rate of cancer recurrence after 5 years ${ }^{(27)}$. Although other studies have indicated that the G. tsugae polysaccharide can enhance splenic NK cell activity and serum interferon production in mice ${ }^{(28)}$, the present study detected no significant difference between the levels of NK cells for the control and experimental groups following treatment.

Chemotherapeutic medicines for treating breast cancer also target $\mathrm{B}$ and $\mathrm{T}$ lymphocytes, which are critical for the adaptive immune response. However, T helper lymphocytes and $\mathrm{CD}^{+}$ cells are especially slow to recover following chemotherapeutic treatments ${ }^{(29)}$. For patients with solid tumours that have achieved high levels of $\mathrm{CD}^{+}$cells during an extended follow-up period, these patients have also been found to experience a longer time to progression ${ }^{(30)}$. In another study by Hakim et $a l^{(29)}$, levels of $\mathrm{CD}^{+}$cells were found to decrease more than $60 \%$ following cancer treatment, and these levels did not return to normal 18 months after treatment was completed. Ferrandina et $a l^{(31)}$ also showed that the recovery of $\mathrm{CD}^{+}, \mathrm{CD}^{+}$and $\mathrm{CD}^{+}$lymphocytes represented independent markers of a longer time to progression, and was also survival markers in ovarian cancer patients that had received high doses of chemotherapy with peripheral blood stem cell and growth factor supplements. In combination, these results suggest that enhancing the recovery of $\mathrm{CD}^{+}$, $\mathrm{CD}^{+}$and $\mathrm{CD}^{+}$lymphocytes not only increases a patient's immunity against infection, but also increases patient survival.

C. pilosula is another commonly used Chinese herbal medicine. The root of $C$. pilosula, of the family Campanulaceae, is beneficial to the immune, digestive and haematopoietic systems. It also induces saliva production, and has been used to treat fatigue, thirst and loss of appetite ${ }^{(32)}$. In a study by Wang et al. ${ }^{(10)}$, C57BL/6 mice that received drinking water containing a polysaccharide-enriched fraction prepared from C. pilosula root exhibited lower mitogenic responses by splenocytes to concanavalin A and lipopolysaccharide. A. sinensis is another herb that has been used in traditional Chinese medicine for the treatment of a wide variety of diseases over thousands of years. Correspondingly, a polysaccharide of A. sinensis has been shown to increase the proliferation of total spleen cells, macrophages and T lymphocytes ${ }^{(11)}$. Furthermore, treatment with this polysaccharide was associated with an increase in the production and expression of IL-2 and interferon- $\gamma$, and a concomitant decrease in IL- 4 expression and production. Flow cytometry assays have also detected an increase in the percentage of $\mathrm{CD}^{+}$lymphocytes in total spleen populations following treatment with the $A$. sinensis polysaccharide ${ }^{(33)}$. In combination, these results demonstrate that Chinese herbal medicine can provide valuable insights into the capacity for modulation of inflammatory cytokine secretion, lymphocyte proliferation and antibody production to improve an individual's immunity. In the present study, it is hypothesised that the maintenance of $\mathrm{T}$ lymphocyte levels associated with RG-CMH may be due to the action of polysaccharides from both C. pilosula and A. sinensis.

In summary, the administration of RG-CMH was associated with an improvement in the conditions of leucopenia and neutropenia experienced by breast cancer patients during treatment. As such, it appears that administration of RG$\mathrm{CMH}$ to patients receiving chemotherapy/radiotherapy would represent a strategy for improving patient immunity. It is hypothesised that the limited sample size of this study did not affect the differences observed between the control and the experimental groups regarding the immune cell counts monitored. However, a larger sample size will be needed to confirm the effect of RG-CMH on lymphocytes and lymphocyte subsets in future studies.

\section{Acknowledgements}

The authors would like to thank all cancer patients who participated in this study. This research received no specific grant from any funding agency in the public, commercial or 
not-for-profit sectors. S. R. Z. and S. L. C. designed the study and revised the manuscript, H. F. C. and J. H. T. conducted the research. M. Y. L. and H. S. L. analysed the data. Y. C. S., Y. Y. Y. and G. T. S. did the laboratory work and prepared the manuscript under the supervision of C.-K. W. All the authors read and approved the final manuscript. None of the authors has any conflicts of interest in the writing and publication of the present study.

\section{References}

1. Karrison T, Ferguson D \& Meier P (1999) Dormancy of mammary carcinoma after mastectomy. J Natl Cancer Inst 91, 80-85.

2. Mafune KI \& Tanaka Y (2000) Influence of multimodality therapy on the cellular immunity of patients with esophageal cancer. Ann Surg Oncol 7, 609-616.

3. Solomayer EF, Feuerer M \& Bai L (2003) Influence of adjuvant hormone therapy and chemotherapy on the immune system analysed in the bone marrow of patients with breast cancer. Clin Cancer Res 9, 174-180.

4. Mccoy JL, Rucker R \& Petros JA (2000) Cell-mediated immunity to tumor-associated antigens is a better predictor of survival in early stage breast cancer than stage, grade, or lymph node status. Breast Cancer Res 60, 227-234.

5. Von Mensdorrl-Pouilly S, Verstraeten AA, Kenemans P, et al. (2000) Survival in early breast cancer patients is favourably influenced by a natural humoral immune response to polymorphic epithelial mucin. J Clin Oncol 18, 547-582.

6. Tagliaferri M, Cohen I \& Tripathy D (2001) Complementary and alternative medicine in early stage breast cancer. Semin Oncol 28, 121-134.

7. Newman V, Rock C \& Faerber S (1998) Dietary supplement use by women at risk for breast cancer recurrence: the Women's Healthy Eating and Living Study Group. J Am Diet Assoc 98, 285-292.

8. Von Gruenigen V, White L \& Kirven M (2001) A comparison of complementary and alternative medicine use by gynecology and gynecologic oncology patients. Int J Gynecol Cancer 11, 205-209.

9. Wasser SP \& Weis AL (1999) Therapeutic effects of substances occurring in higher Basidiomycetes mushrooms: a modern perspective. Crit Rev Immunol 19, 65-96.

10. Wang ZT, Ng TB, Yeung HW, et al. (1996) Immunomodulatory effect of a polysaccharide-enriched preparation of Codonopsis pilosula roots. Gen Pharmacol 27, 1347-1350.

11. Hardy ML (2000) Herbs of special interest to women. J Am Pharm Assoc 40, 234-242.

12. Bradley RR, Cunniff PJ \& Pereira BJ (1999) Hematopoietic effect of Radix Angelicae sinensis in a hemodialysis patient. Am J Kidney Dis 34, 349-354.

13. Sun W, Xu Z \& Wang C (2005) Study on antioxidant activity of essential oils and its monomer from Pelargonium graveolens. Zhong Yao Cai 28, 87-89.

14. Tong AW, Seamour B, Lawson JM, et al. (2000) Cellular immune profile of patients with advanced cancer before and after taxane treatment. Am J Clin Oncol 23, 463-472.

15. Gan KH, Fann YF, Hsu SH, et al. (1998) Mediation of the cytotoxicity of lanostanoids and steroids of Ganoderma tsugae through apoptosis and cell cycle. J Nat Prod 61, 485-487.

16. Jinn TR, Wu CM, Tu WC, et al. (2006) Functional expression of FIBP-gts, a fungal immunomodulatory protein from
Ganoderma tsugae in Sf21 insect cells. Biosci Biotechnol Biochem 70, 2627-2634

17. Kino K, Yamashita A \& Yamaoka K (1989) Isolation and characterization of a new immunomodulatory protein, ling zhi-8 (LZ-8), from Ganoderma lucidium. J Biol Chem 264 , $472-478$.

18. Ko JL, Hsu CI, Lin RH, et al. (1995) A new fungal immunomodulatory protein, FIP-fve isolated from the edible mushroom, Flammulina velutipes, and its complete amino acid sequence. Eur J Biochem 228, 244-249.

19. Hsu HC, Hsu CI, Lin RH, et al. (1997) Fip-vvo, a new fungal immunomodulatory protein isolated from Volvariella volvacea. Biochem J 323, 557-565.

20. Lin WH, Hung CH, Hsu CI, et al. (1997) Dimerization of the N-terminal amphipathic alpha-helix domain of the fungal immunomodulatory protein from Ganoderma tsugae defined by a yeast two-hybrid system and site-directed mutagenesis. J Biol Chem 272, 20044-20048.

21. Anonymous (2002) Creating a neutropenia risk model that really works. ADVANCE Awareness Neutropenia Chermother 1, 1-6.

22. Balducci L \& Yates J (2000) General guidelines for the management of older adults with cancer. Oncology 14, 221-227.

23. Herberman RB \& Ortaldo JR (1981) Natural killer cells: their role in defense against disease. Science 214, 24-30.

24. Minato N, Bloom BR, Jones C, et al. (1979) Mechanism of rejection of virus persistently infected tumor cells by athymic mice. J Exp Med 149, 1117-1133.

25. Locke S, Kraus L, Leserman J, et al. (1984) Life change stress, psychiatric symptoms and natural killer cell activity. Psychosom Med 46, 441-453.

26. Brittenden J, Heys S, Ross J, et al. (1996) Natural killer cells and cancer. Cancer 77, 1226-1243.

27. Levy S, Herberman R, Lippman M, et al. (1991) Immunological and psychosocial predictors of disease recurrence in patients with early-stage breast cancer. Behav Med 17, $67-75$.

28. Won SJ, Lin MT \& Wu WL (1992) Ganoderma tsugae mycelium enhances splenic natural killer cell activity and serum interferon production in mice. Jpn J Pharmacol 59, $171-176$.

29. Hakim FTR, Cepeda S \& Kaimei S (1997) Constraints on CD4 recovery post chemotherapy in adults: thymic insufficiency and apoptotic decline of expanded peripheral CD4 cells. Blood 90, 3789-3798.

30. Pierelli L, Perillo A, Ferrandina G, et al. (2001) The role of growth factor administration and T-cell recovery after peripheral blood progenitor cell transplantation in the treatment of solid tumors: results from a randomized comparison of G-CSF and M-CSF. Transfusion 41, 1577-1585.

31. Ferrandina G, Pierelli L, Perillo A, et al. (2003) Lymphocyte recovery in advanced ovarian cancer patients after highdose chemotherapy and peripheral blood stem cell plus growth factor support: clinical implications. Clin Cancer Res 9, 195-200.

32. Wang ZT, Du Q, Xu GJ, et al. (1997) Investigations on the protective action of Codonopsis pilosula (Dangshen) extract on experimentally-induced gastric ulcer in rats. Gen Pharmacol 28, 469-473.

33. Yang T, Jia M, Meng J, et al. (2006) Immunomodulatory activity of polysaccharide isolated from Angelica sinensis. Int J Biol Macromol 39, 179-184. 